\title{
Evaluating the efficacy and safety of the carotid CASPER-RX stent in mechanical thrombectomy for acute ischemic stroke
}

\author{
B. Bartolini, F. Puccinelli, P.J. Mosimann, S.D. Hajdu, L. Veunac, P. Michel, G. Saliou
}

A new generation of carotid artery stents that uses a dual micromesh layer to reduce embolic events during carotid artery stenting has recently been introduced. We aim to analyze the efficacy and safety of the new CASPER-RX stent in patients experiencing acute ischemic stroke with large vessel intracranial occlusion associated with a tandem lesion (i.e. also a carotid occlusion or severe stenosis).

All consecutive patients treated with carotid CASPER-RX stents were retrospectively analyzed from our stroke registry. Clinical, angiographic and neuroimaging data were analyzed. Endpoints included acute intra-stent thrombus formation, stent occlusion prior to hospital discharge, 3-month modified Rankin score (mRs) and symptomatic intracranial hemorrhage.

Twenty-one patients were included. Ten patients $(47,6 \%)$ had tandem carotid occlusions and eleven patients had severe carotid stenosis (52,4\%), eight of whom had a haemodynamically significant stenosis. Fifteen patients had M1 (71,4\%) intracranial occlusion and six patients had M2 (18,6\%) intracranial occlusion. All 21 patients were loaded with 250-500mg aspirin intravenously. Additionally, 18 of these patients received intravenous r-tPA and four patients were already taking $75 \mathrm{mg}$ clopidogrel daily. Seven patients received a bolus of heparin intravenously (range 3000-6000 units). No stent occlusion occurred prior to hospital discharge. Symptomatic intracranial hemorrhage was reported in two patients. No stroke recurrence was reported.

Baseline Characteristic and Medical History

\begin{tabular}{|l|c|}
\hline Age (mean \pm SD ) & $71,3 \pm 9,8$ \\
\hline Female, \% & 38,1 \\
\hline Smoking, \% & 38,1 \\
\hline Hypertension, \% & 71,4 \\
\hline Dislipidemia, \% & 90,5 \\
\hline Atrial Fibrillation, \% & 9,5 \\
\hline Diabetes Mellitus, \% & 14,3 \\
\hline $\begin{array}{l}\text { Baseline NIHSS (median, } \\
\text { [IQR]) }\end{array}$ & 15 [9,5-19] \\
\hline IV-tPA, \% & 85,7 \\
\hline
\end{tabular}

Post stent findings

\begin{tabular}{|l|c|}
\hline Stent length, mean $\pm S D, m m$ & $30,2 \pm 2,2$ \\
\hline $\begin{array}{l}\text { Stent diameter, } \\
\text { mean } \pm S D, m m\end{array}$ & $7,2 \pm 1,4$ \\
\hline $\begin{array}{l}\text { Acute intra-stent thrombus } \\
\text { formation, } \mathrm{n}(\%)\end{array}$ & $11(52,4)$ \\
\hline $\begin{array}{l}\text { Incomplete vessel wall } \\
\text { apposition, } \mathrm{n}(\%)\end{array}$ & $1(4,8)$ \\
\hline $\begin{array}{l}\text { Residual stenosis, } \\
\text { mean } \pm S D, \%\end{array}$ & $16,5 \pm 11,2$ \\
\hline Single Stent $\mathrm{n}(\%)$ & $18(86)$ \\
\hline Multiple Stents $\mathrm{n}(\%)$ & $3(14)$ \\
\hline
\end{tabular}

\section{0-days MRs}

$19,0 \% \quad 42,9 \% \quad 9,5 \% \quad 9,5 \% 4,8 \% \quad 14,3 \%$

MRs 0-2: $71,4 \%$

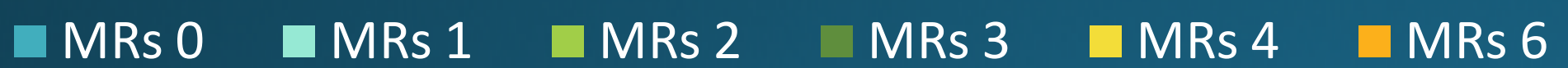

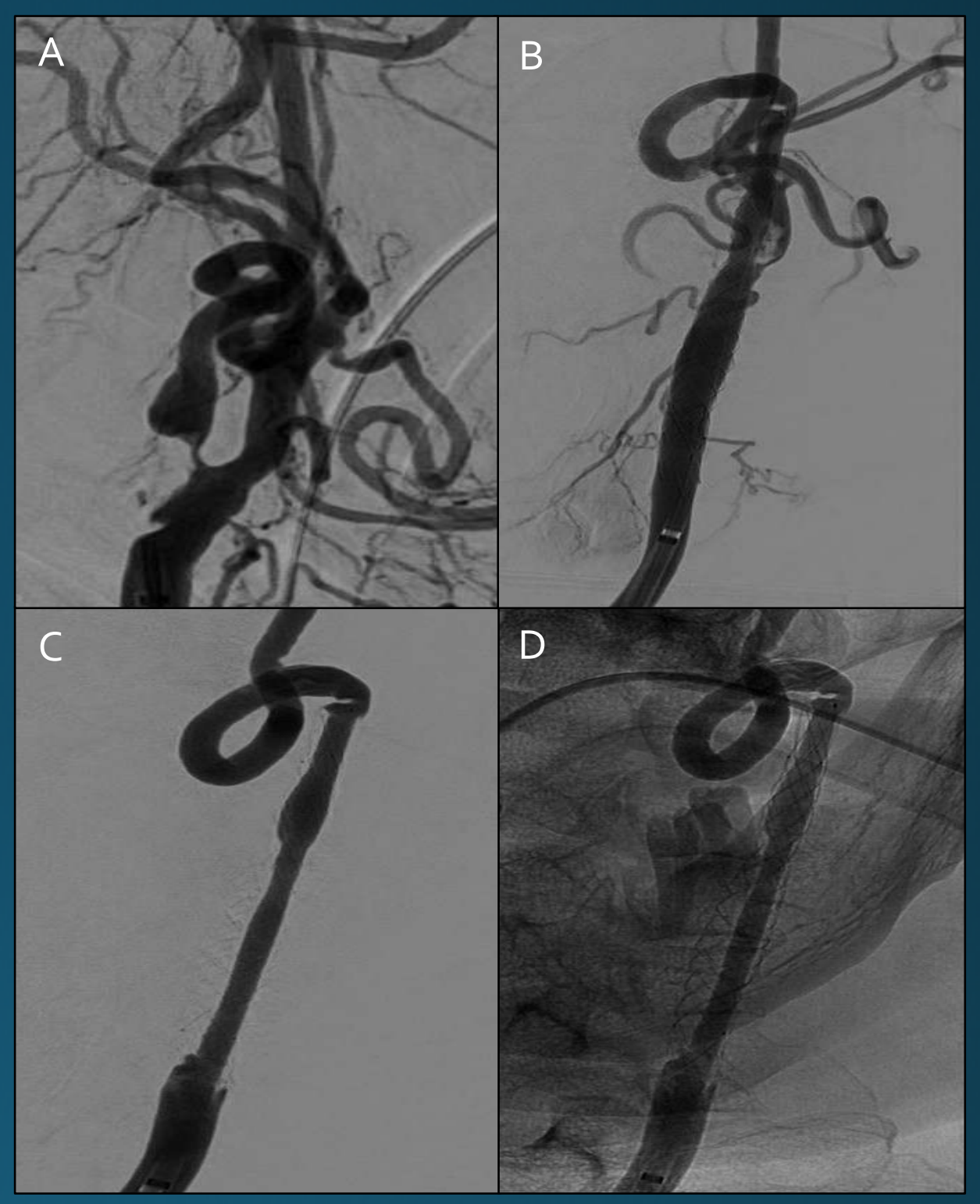

A: substracted DSA: pre-stent carotid stenosis B: substracted DSA: immediate post-stent control C: substracted DSA: delayed post-stent control, acute intra-stent thrombus

D: unsubstracted DSA: delayed post-stent control, acute intra-stent thrombus

A recent article found a significantly higher rate of acute stent occlusions of dual-layer carotid artery stents compared with singlelayer stents in the setting of acute stroke with an intracranial occlusion and an additional occlusion at the origin of the ICA (tandem occlusions).*

In the present series, clot formation during the procedure was frequently observed in Casper-RX stents, which required repeated balloon angioplasty, peri-procedural intravenous infusion of anticoagulant and antiplatelet treatment in addition to $r$-tPA. In the absence of a prospective randomized controlled study demonstrating the non-inferiority of micromesh dual-layer stent compared to single-layer design, we decided to no longer use this stent in acute stroke when carotid angioplasty was also required.

* Yilmaz, U., Körner, H., Mühl-Benninghaus, R., Simgen, A., Kraus, C., Walter, S., et al. (2017). Acute Occlusions of Dual-Layer Carotid Stents After Endovascular Emergency Treatment of Tandem Lesions. Stroke; a Journal of Cerebral Circulation, 48(8), 2171-2175. http://doi.org/10.1161/STROKEAHA.116.015965 